\title{
Una actividad de aprendizaje específica de construcciones del triángulo con el uso del Software Geogebra
}

\author{
Norma Gutiérrez¹, Angélica G. Villegas', Claudia G. Pavano', Claudia Flores² \\ ${ }^{1}$ Academia de Matemáticas -Instituto Politécnico Nacional - CECyT 10, Ciudad de \\ México, México \\ ${ }^{2}$ Academia de Matemáticas -Instituto Politécnico Nacional - CECyT 5, Ciudad de \\ México, México
}

ngutierrez@ipn.mx, agvillegas@ipn.mx, cpavano@ipn.mx,cfloreses@ipn.mx

\begin{abstract}
The purpose of this work is showing the results of the research project "Implementation of learning activities as a teaching strategy in the teaching-learning of triangles in high school". It was designed and implemented over a series of learning activities with the use of dynamic software GeoGebra. It is expected that by these activities the student will recognize the elements and properties of triangles, which are intended to redefine and restructure teaching and learning processes aimed at developing skills. Otherwise, using the Theory of Teaching Situations as a reference, considering what the teacher wants to promote in the construction of knowledge.

Resumen. El propósito de este trabajo es mostrar los resultados del proyecto de investigación "Implementación de actividades de aprendizaje como estrategia didáctica en la enseñanza-aprendizaje de triángulos en el nivel medio superior". Se elaboró el diseño y se implementó una serie de actividades de aprendizaje con el uso del software dinámico Geogebra; se espera que con estas actividades el alumno reconozca los elementos y propiedades de los triángulos, mismas con las que se pretende redefinir y reestructurar los procesos de enseñanza y aprendizaje tendientes al desarrollo de competencias. Además de usar como referencia la Teoría de las Situaciones Didácticas, considerando lo que hace el docente para propiciar la construcción del conocimiento.
\end{abstract}

\section{Introducción}

En Zaldívar, J., Medina, G. y Kakes, A. (2018), señala que la investigación en Matemática Educativa cada vez se enfrenta al surgimiento de nuevas tecnologías potenciales para su integración en el aula de matemáticas, proporcionando un cambio en la enseñanza y el aprendizaje de la misma.

Por otro lado, sabemos que hay un cambio radical en la educación. La educación 4.0 ofrece la gran ventaja de poder ser adaptada a las necesidades e intereses de los estudiantes en virtud de las exigencias de la industria. Una característica de ésta es que 
proporciona un conocimiento técnico y digital, para que nuestros alumnos se puedan desarrollar en cualquier ámbito.

Es por ello, que con el uso de la tecnología se analizan las cuestiones educativas para tomar en cuenta el contexto para el diseño e implementación de las actividades de aprendizaje.

En este proyecto se diseñaron e implementaron una serie de actividades de aprendizaje con el uso de un software dinámico interactivo: Geogebra, el cual nos permitió observar a los estudiantes enfrentarse a estas actividades y reconocer si era esencial la realización de las mismas para que ellos pudieran por medio de la visualización comprobar las propiedades de los triángulos y así nosotros como docentes hacer una reestructuración en nuestra enseñanza.

Si nos referimos a la evaluación del aprendizaje, es importante considerar todos los elementos incluidos, no solo el trabajo de los estudiantes, sino también su necesidad al uso de la tecnología, el desempeño del profesor, la eficiencia de los materiales, el ambiente e incluso el contexto de la actividad.

\section{Marco teórico}

El marco teórico lo ubicamos en la importancia del estudio del currículo para la mejora del sistema educativo. Desde el marco de los currículos, el currículo formal es la planeación del proceso de enseñanza-aprendizaje basado en el programa académico; los programas de un plan de estudio consideran los objetivos generales y particulares de aprendizaje; competencia general y competencias particulares.

El Currículo Potencialmente Aplicado (CPA) que ofrecemos para el área de matemáticas se organiza a través de redes de actividades. Suárez y et al (2012) mencionan que el CPA puede comprender materiales (paquetes didácticos), planes (de seguimiento, capacitación y evaluación) y dispositivos organizacionales (redes y comunidades, con un marco de operación explícito) que concretan el currículo planeado desde una perspectiva de sistema y profesional.

La presente investigación encuentra su marco de referencia en la Teoría de Situaciones Didácticas e Ingeniería Didáctica. Su interés principal se enfoca en el estudio y diseño de ingenierías didácticas para matemáticas, en busca de favorecer el desarrollo de habilidades y la adquisición de saberes por parte del alumno en un esquema experimental basado en las realizaciones didácticas en clase, es decir, sobre la concepción, realización, observación y análisis de situaciones didácticas basadas en la confrontación entre el análisis a priori y a posteriori. (Artigue, 1995).

\section{Metodología}

Como parte de un proyecto de investigación, se planteó la aplicación del diseño de una serie de actividades de aprendizaje, con la finalidad de mejorar la enseñanza de la Unidad de Aprendizaje de Geometría y Trigonometría y de Geometría Analítica. La pregunta de 
VIII Congresso Brasileiro de Informática na Educação (CBIE 2019)

Anais dos Workshops do VIII Congresso Brasileiro de Informática na Educação (WCBIE 2019)

investigación es ¿Cuál es el nivel de aprendizaje que logran los estudiantes con este tipo de actividades?

Para la implementación de estas actividades, se llevó a cabo un taller con docentes y alumnos dentro de nuestra institución. El taller para los docentes y alumnos, se llevó a cabo en la sala siglo XXI y el aula de cómputo en las instalaciones del CECyT 10 del Instituto Politécnico Nacional respectivamente.

En la primera sesión del Taller, tanto a los profesores de matemáticas como a los alumnos, se les explica de manera general las herramientas con las que cuenta el software Geogebra para el desarrollo de las actividades, que ellos con sus construcciones del triángulo puedan demostrar por medio de la visualización las propiedades y elementos de los triángulos y así como también se explica la modalidad de trabajo.

Las siguientes sesiones consisten en que ellos realicen de dos a cuatro actividades, donde se requiere el uso del software Geogebra, las cuales están planeadas poniendo en ellas la forma de construcción y así puedan verificar las propiedades con sus construcciones individuales. En estas prácticas se desarrollan las comprobaciones de las propiedades:

- La suma de los ángulos interiores de un triángulo es igual a $180^{\circ}$.

- La suma de los ángulos externos es igual a $360^{\circ}$.

- Todo triángulo equilátero, es decir las medidas de sus ángulos internos son iguales.

- Trazar y encontrar la ecuación de la mediana de un triángulo.

- Trazar y determinar la ecuación de la mediatriz de un triángulo

Para analizar la información se consideran algunos instrumentos alternativos de evaluación en particular la tabla de resultados y la lista de cotejo, como se describen en Flores y Gómez (2009) en el que consideran la pregunta de la actividad de aprendizaje, la respuesta esperada, la respuesta obtenida por parte del estudiante y las observaciones.

\section{Resultados}

En la fase de planeación hay que considerar la caracterización (Tabla 1) de algunas de las actividades de aprendizaje (AIM-NMS-IPN, 2002), ésta permite organizar la información, clasificar las actividades e identificar si el problema o actividad de aprendizaje tiene el potencial para lograr la competencia en el estudiante como lo mencionan en Flores y Gómez (2009).

Para conformar y caracterizar la red de actividades que el estudiante realizará en la red de aprendizaje se consideraron las siguientes: 
VIII Congresso Brasileiro de Informática na Educação (CBIE 2019)

Anais dos Workshops do VIII Congresso Brasileiro de Informática na Educação (WCBIE 2019)

Tabla 1. La caracterización de la actividad

\begin{tabular}{|l|l|}
\hline \multicolumn{2}{|c|}{ Caracterización de la actividad } \\
\hline Actividad de aprendizaje & Ejercicio, problema o problema con guía \\
\hline Modalidad de trabajo & Individual o equipo \\
\hline Lugar de realización & Salón de clases, aula de cómputo \\
\hline Herramientas tecnológicas & Software, calculadora científica, dispositivo móvil \\
\hline Referencias curriculares & Programa Académico \\
\hline Tiempo & 1 hora \\
\hline Producto & $\begin{array}{l}\text { Reporte por escrito de la actividad o por envío a un correo } \\
\text { electrónico }\end{array}$ \\
\hline Representaciones & Algebraica, gráfica. \\
\hline Estrategias & $\begin{array}{l}\text { Organiza la información } \\
\text { Analiza un caso particular }\end{array}$ \\
\hline Evaluación & Rúbrica \\
\hline
\end{tabular}

\subsection{Implementación con los docentes}

La puesta en escena de las actividades de aprendizaje se llevó a cabo con seis profesores $\left(\mathrm{P}_{\mathrm{i}}\right)$ de matemáticas de la Academia de Matemáticas del turno matutino en el CECyT 10, los resultados obtenidos en la actividad de aprendizaje propiedad 6: "La suma de los ángulos externos es igual a $360^{\circ}$ " se dan a través de la siguiente Lista de cotejo 1.

Lista de cotejo 1. "La suma de los ángulos externos es igual a $360^{\circ}$ ”

\begin{tabular}{|l|c|c|c|c|c|c|}
\hline & $\mathrm{P}_{1}$ & $\mathrm{P}_{2}$ & $\mathrm{P}_{3}$ & $\mathrm{P}_{4}$ & $\mathrm{P}_{5}$ & $\mathrm{P}_{6}$ \\
\hline Abre y entiende el graficador & $*$ & $*$ & $*$ & $*$ & $*$ & $*$ \\
\hline Traza un triángulo & $*$ & $*$ & $*$ & $*$ & $*$ & $*$ \\
\hline Traza las semirectas desde los vértices & $*$ & $*$ & $*$ & $*$ & $*$ & $*$ \\
\hline Traza los ángulos externos & & $*$ & & & & $*$ \\
\hline Mide los ángulos externos & $*$ & $*$ & $*$ & $*$ & $*$ & $*$ \\
\hline Suma los ángulos externos & & $*$ & & $*$ & & $*$ \\
\hline La suma de los ángulos externos es igual a 360 & $*$ & $*$ & $*$ & $*$ & $*$ & \\
\hline Comprobación de la propiedad & $*$ & $*$ & $*$ & $*$ & $*$ & $*$ \\
\hline
\end{tabular}

En esta lista de cotejo se puede observar que más de la mitad de los profesores realizaron los trazos correspondientes mediante el uso de Software Geogebra considerando las propiedades, ángulos y teoremas del triángulo. 
VIII Congresso Brasileiro de Informática na Educação (CBIE 2019)

Anais dos Workshops do VIII Congresso Brasileiro de Informática na Educação (WCBIE 2019)

En la tabla 2 se muestran las respuestas de los profesores a la actividad diseñada de acuerdo a los puntos señalados en la lista de cotejo 1 .

Tabla 2. Resultados de la Actividad de Aprendizaje. Elaboración propia.

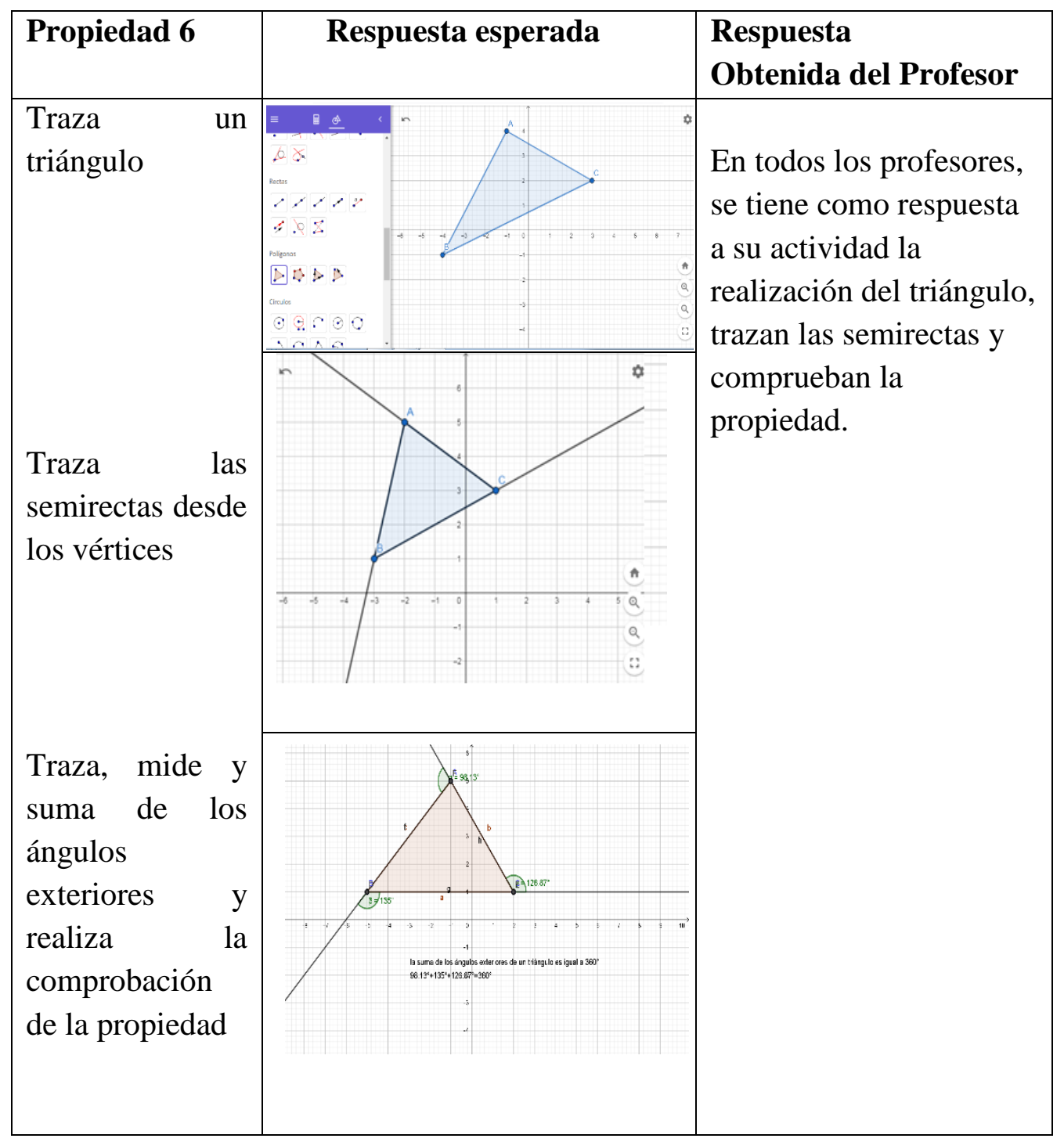

\subsection{Implementación con los alumnos de primer semestre}

El desarrollo de las actividades para los alumnos de primer semestre, se realiza posterior al de los docentes, de acuerdo a las retroalimentaciones de dichas actividades, se realizan los ajustes necesarios; por otro lado, para conformar y caracterizar la red de actividades que el estudiante realizara se consideró la misma caracterización del docente.

La primera implementación de prácticas se llevó a cabo con los alumnos de primer semestre donde ellos verifican algunas de las propiedades de los triángulos (ángulos interiores de cualquier triángulo y de un triángulo equilátero) y posteriormente se realizó otra práctica con alumnos de tercer semestre donde construyen y definen el lugar geométrico de una línea (mediana) de todo triángulo. 
VIII Congresso Brasileiro de Informática na Educação (CBIE 2019)

Anais dos Workshops do VIII Congresso Brasileiro de Informática na Educação (WCBIE 2019)

La puesta en escena de ésta actividad fue con 16 estudiantes de primer semestre que cursan la unidad de aprendizaje de Álgebra y se llevó a cabo en el aula de cómputo Siglo XXI en el CECyT 10.

Se considera que nuestros alumnos ya cuentan con conocimientos previos, en la Escuela Básica tuvieron el aprendizaje de la figura "triángulo" en relación a sus propiedades y rectas notables. En esta investigación se hizo la secuencia de dos actividades de aprendizaje, el profesor no interactúa con ellos, ya que las secuencias están diseñadas de tal manera que trabajen solos.

Con ellos se lleva a cabo la comprobación de las propiedades:

1. La suma de los ángulos interiores de un triángulo es igual a $180^{\circ}$.

2. Todo triángulo equilátero, es decir las medidas de sus ángulos internos son iguales.

Los resultados de los alumnos de primer semestre, en su mayoría son parecidos, por lo que en la Lista de cotejo 2, sólo se muestran los de dos alumnos, y como se verifica en dicha lista, los alumnos realizan la actividad sin ningún problema, ya que en general, desarrollan cada una de las instrucciones solicitadas.

Lista de cotejo 2. "La suma de los ángulos internos es igual a $180^{\circ}$ "

\begin{tabular}{|l|c|c|}
\hline & $\mathrm{P}_{1}$ & $\mathrm{P}_{2}$ \\
\hline Abre y entiende el graficador & $*$ & $*$ \\
\hline Traza un triángulo & $*$ & $*$ \\
\hline Traza los ángulos internos & $*$ & $*$ \\
\hline Mide los ángulos internos & $*$ & $*$ \\
\hline Suma los ángulos internos & $*$ & $*$ \\
\hline La suma de los ángulos internos es igual a $180^{\circ}$ & $*$ & $*$ \\
\hline Comprobación de la propiedad & $*$ & $*$ \\
\hline
\end{tabular}

\subsection{Implementación con los alumnos de tercer semestre}

En el caso de esta actividad, la puesta en escena fue con un grupo de 45 alumnos de tercer semestre que cursan la unidad de aprendizaje de Geometría Analítica en un aula de clases.

Considerando que estos alumnos ya tienen los elementos suficientes para determinar la ecuación general de una recta a partir de dos condiciones dadas, un punto y la pendiente o dos puntos. En esta sesión se hizo uso de dispositivos móviles (celular y tablet), en donde el docente fue dando instrucciones al mismo tiempo que los alumnos, reunidos en equipos, pero trabajando de manera individual, realizaban la construcción. 
VIII Congresso Brasileiro de Informática na Educação (CBIE 2019)

Anais dos Workshops do VIII Congresso Brasileiro de Informática na Educação (WCBIE 2019)

La actividad realizada con los alumnos fue trazar y determinar las medianas de un triángulo, así mismo obtener las coordenadas del baricentro. Al igual que en la actividad anterior, los resultados son similares en todos los alumnos, por lo que solo se muestran los resultados de dos de ellos en la Lista de cotejo 3.

Lista de cotejo 3. "Traza y determina la ecuación de las medianas de un triángulo"

\begin{tabular}{|l|c|c|}
\hline & $\mathrm{P}_{1}$ & $\mathrm{P}_{2}$ \\
\hline Abre y entiende el graficador & $*$ & $*$ \\
\hline $\begin{array}{l}\text { Ingresa correctamente las coordenadas de los } \\
\text { vértices del triángulo }\end{array}$ & $*$ & $*$ \\
\hline Traza el triángulo & $*$ & $*$ \\
\hline $\begin{array}{l}\text { Determina los puntos medios de los lados del } \\
\text { triángulo }\end{array}$ & $*$ & $*$ \\
\hline $\begin{array}{l}\text { Traza las medianas y determina las coordenadas } \\
\text { del baricentro del triángulo }\end{array}$ & $*$ & $*$ \\
\hline
\end{tabular}

Los resultados de ambas actividades que se aplicaron con alumnos tanto de primer como de tercer semestre son interesantes, porque aparte de generar la figura, el trazo de los ángulos interiores, así como las coordenadas del baricentro del triángulo, respaldan las actividades con sus resultados elaborados en clase a lápiz y papel, como se muestra en la Tabla 3.

Tabla 3. Resultados de la Actividad de Aprendizaje. Elaboración propia.

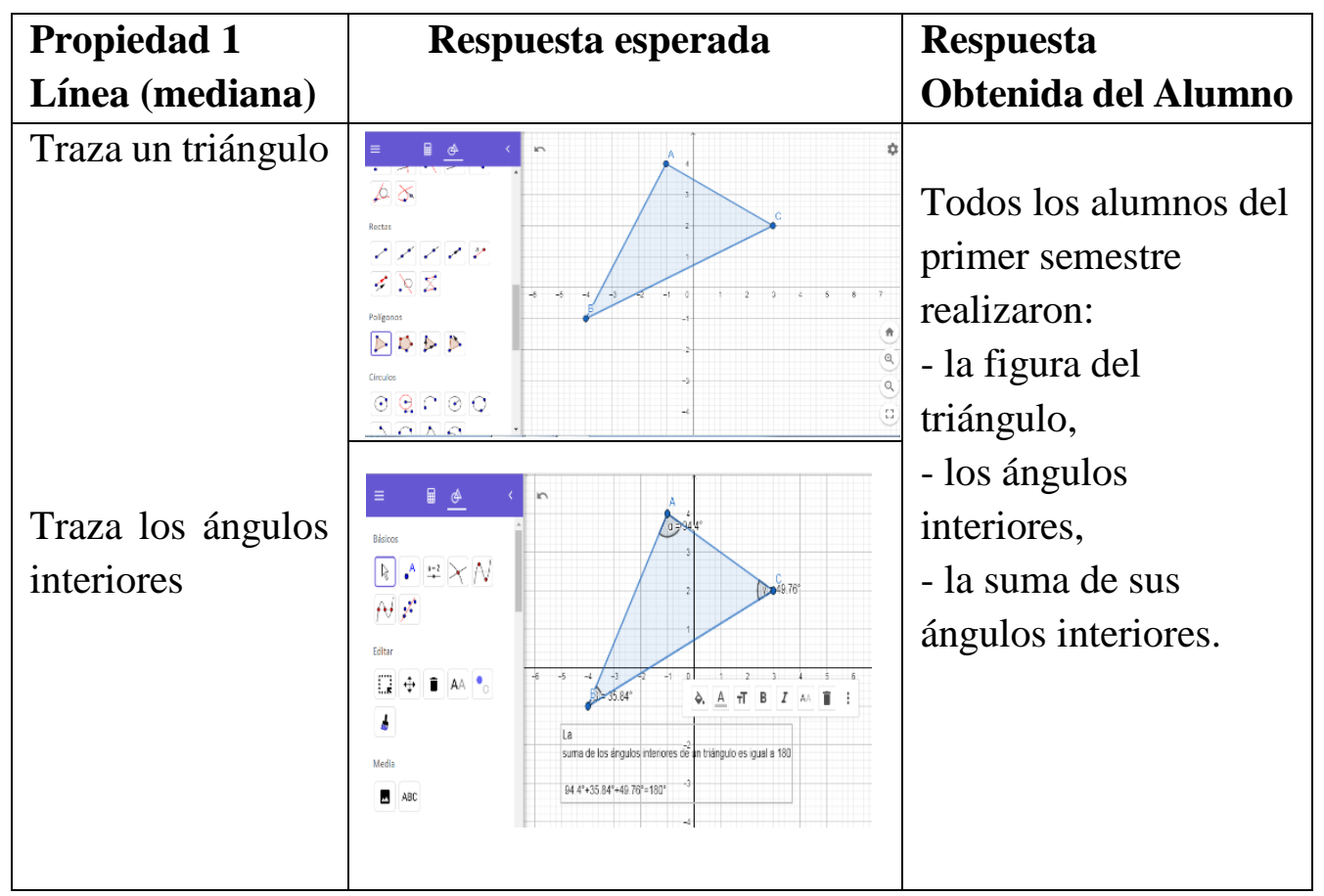


VIII Congresso Brasileiro de Informática na Educação (CBIE 2019)

Anais dos Workshops do VIII Congresso Brasileiro de Informática na Educação (WCBIE 2019)

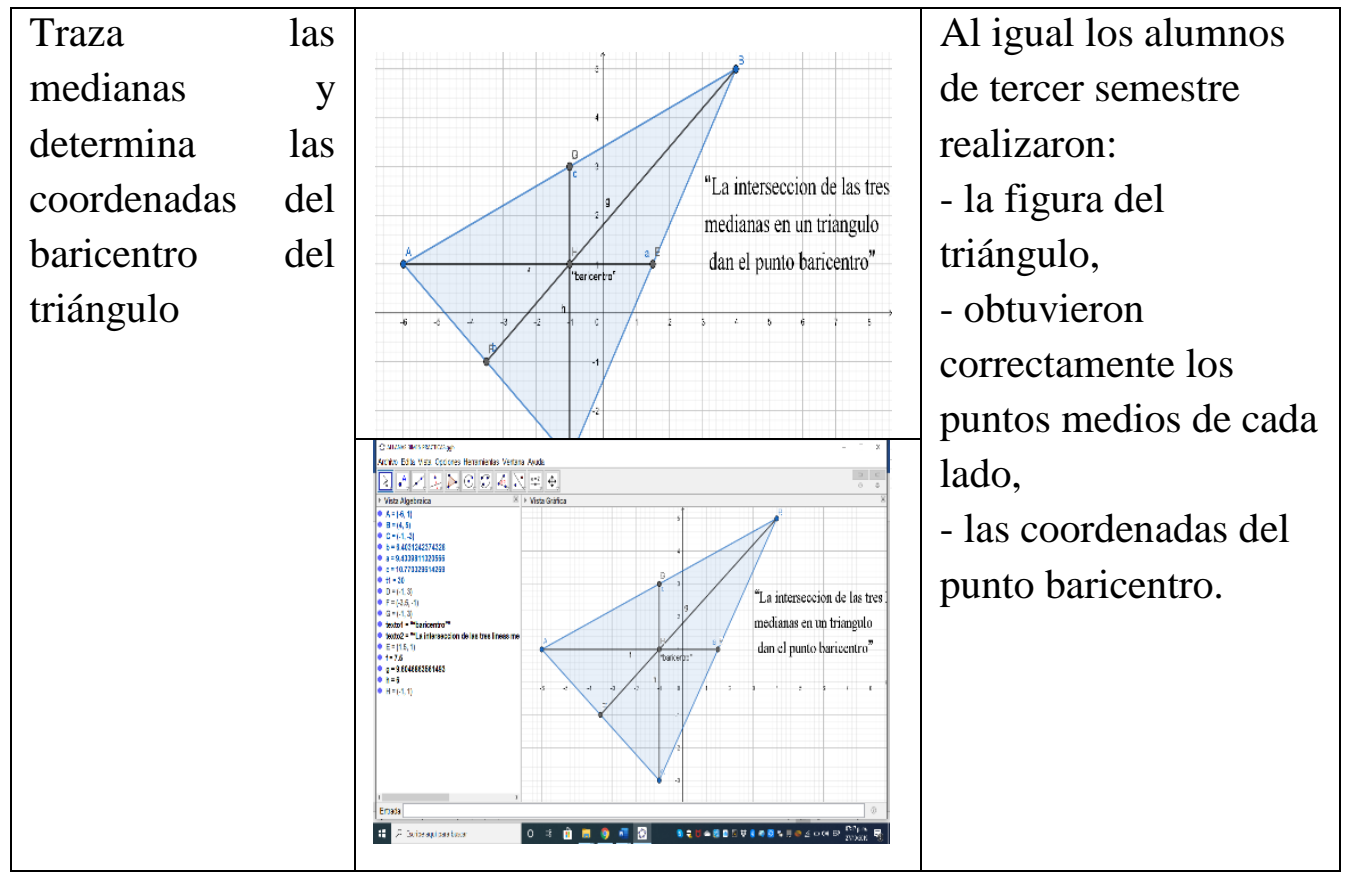

\section{Conclusiones}

En el uso del software interactivo Geogebra tanto para los profesores y los estudiantes se mostró interés en la resolución de las actividades, ya que no se trata de que ellos sepan a profundidad el manejo del software, éste software los lleva de la mano con sus indicaciones y el diseño de sus comandos, aparte de que las actividades están elaboradas con instrucciones muy fáciles de seguir y se percatan que estas actividades refuerzan lo aprendido en relación a las propiedades de los triángulos.

En este taller los profesores comentaron que las actividades pueden llevarse a cabo con estudiantes de segundo o tercer semestre, considerando que se debe enseñar conocimientos previos en el momento de implementarlos con los estudiantes. Las actividades diseñadas en el material didáctico se consideran pertinentes e interesantes para implementarlos en el aula de clases, usando el software que ayude a contrastarlo con lo realizado a lápiz y papel.

Sin embargo, con los alumnos fueron sesiones rápidas, ya que no se les dificulta el manejo de la tecnología, así como tampoco el manejo del software, para ellos fue muy fácil poder realizar las actividades, además, es importante destacar la iniciativa de los alumnos por explorar en el software, ya que la mayoría de ellos le dio formato personalizado a la construcción realizada cambiando principalmente los colores, con lo que podemos concluir que es una práctica atractiva para ellos.

La tabla de resultados nos permite ver tanto los logros que se tienen por parte de los docentes como de los alumnos, además de considerar que para una etapa posterior se deberá instruir a los profesores para que la utilicen con los estudiantes comparando los resultados que ellos esperaban, con los obtenidos en realidad, dándonos a nosotros como 
VIII Congresso Brasileiro de Informática na Educação (CBIE 2019)

Anais dos Workshops do VIII Congresso Brasileiro de Informática na Educação (WCBIE 2019)

investigadores, la oportunidad de comparar los resultados esperados al planear la actividad, los que esperaban los profesores y lo que en realidad sucedió con los estudiantes.

El instrumento que muestra la construcción de conocimientos matemáticos lograda en los estudiantes es la lista de cotejo la cual nos permite ver los puntos específicos que hicieron y, bien elegidos, pueden mostrar los logros o los tropiezos de los estudiantes al implementar estas actividades en el aula.

La construcción de conocimientos de los estudiantes al resolver la red de aprendizaje con el uso de las TIC da una mayor posibilidad de analizar diferentes casos, de entender las propiedades y teoremas del polígono triángulo.

La opinión de estos alumnos al usar esta estrategia didáctica es:

- Lilian: ..."Se nos facilita el realizar los ejercicios, porque es más fácil y práctico, es una muy buena opción para entender y al realizar los ejercicios. Nos damos cuenta de cómo se puede medir, realizar figuras y trazos fácilmente"...

- Luis: ..."Me parece una forma entretenida y muy buena para nuestro aprendizaje ya que, utilizando diferentes herramientas, nos aporta aprendizajes que nos podrían servir en nuestros estudios"...

- Jorge: ..."Yo creo que esta actividad fue muy interesante y didáctica para nosotros ya que aprendimos a usar una aplicación que facilitó la graficación. Fue fácil y divertido trabajar en equipo"...

- Axel: ..."Creo que fue una manera muy innovadora de hacer la actividad, esto se debería hacer más seguido"...

Tener un material didáctico desarrollado para unir el Curriculum Institucional con el Curriculum Logrado, apoya al profesor en su labor, pero tenerlo organizado en redes permite que los diferentes aprendizajes se vayan ligando y organizando de manera que su aprendizaje sea más fácil y eficiente al apoyarse en otros conocimientos relacionados y al cobrar significado con el contexto del estudiante, sin importar si se basa en situaciones reales o imaginarias.

\section{Referencias}

AIM (2001). AIM-NMS-IPN. Academia Institucional de Matemáticas. Instituto Politécnico Nacional. México.

Artigue, M. (1995). Ingeniería Didáctica. En Pedro Gómez (Ed.), Ingeniería didáctica en educación matemática. pp. 33-59.

Flores, H. y Gómez, A. (2009). Aprender Matemática, Haciendo Matemática: la evaluación en el aula. Educación Matemática. Vol. 21, no.2, pp. 117-142. 
VIII Congresso Brasileiro de Informática na Educação (CBIE 2019)

Anais dos Workshops do VIII Congresso Brasileiro de Informática na Educação (WCBIE 2019)

IPN (2014). Programa de estudios de la Unidad de Aprendizaje: Geometría Analítica. Secretaría Académica. Dirección de Educación Media Superior. IPN. México.

IPN (2014). Programa de estudios de la Unidad de Aprendizaje: Geometría y Trigonometría. Secretaría Académica. Dirección de Educación Media Superior. IPN. México.

Suárez, L.; Torres, J.L.; Ortega, P. (2012). Las matemáticas del bachillerato en el instituto politécnico nacional. En C. Dolores. (Ed.) ¿Hacia dónde reorientar el currículum de matemáticas del Bachillerato? México: Plaza y Valdés.

Zaldívar, J., Medina, G. y Kakes, A. (2018). Modelación y Tecnología en la enseñanza de las matemáticas. Acta Latinoamericana de Matemática Educativa. Vol. 31, pp. 954961. 